\title{
Mode folding in systems with local interaction: unitary and non-unitary transformations using tensor states
}

\author{
Jose Reslen ${ }^{1}$ \\ ${ }^{1}$ Coordinación de Física, Universidad del Atlántico, \\ Kilómetro 7 Antigua vía a Puerto Colombia, A.A. 1890, Barranquilla, Colombia.
}

\begin{abstract}
An approach to the simulation of locally interacting systems is demonstrated and assayed. The proposal is built upon the concept of folding of bosonic modes previously introduced in the context of linear dynamics and can be seen as an alternative to Trotter-Susuki expansion in studies of quantum propagation based on tensor states. It is shown that evolution as well as ground state computations can be implemented and that test simulations deliver comparatively accurate results. The whole analysis provides insight into the way well-known quantum precursors affect mean values and fluctuations in realistic setups.
\end{abstract}

PACS numbers: 05.30.Jp, 05.10.Cc, 02.70.

\section{INTRODUCTION}

The study of numerical methods of quantum mechanics has become an exciting and increasingly active field of research, not only because numerical methods are extremely important as simulation instruments, but also because they help understand the underlying functioning of the quantum theory. Such is the case of Density Matrix Renormalization Group (DMRG), which has played an important role in this sense since its inception. Time Evolving Block Decimation (TEBD) has appeared more recently and integrates elements of DMRG with concepts of quantum computation. Both DMRG and TEBD have inspired a number of variants such as tDMRG, iTEBD, MPS, MERA and PEPS, to mention just a few [1, 2]. However, there exist instances where the lack of efficient simulation protocols is yet an issue. Here, a proposal in this direction is explored, namely, a method that can be used to simulate real as well as imaginary quantum evolution under more relaxed conditions than those encountered in standard TEBD applications. This paper expands on the themes first addressed in reference [3], and can be seen as its continuation, especially in what concerns to the inclusion of interaction and the treatment of non-unitary transformations.

Many of the efforts devoted during recent times to the study of efficient simulation options have been motivated by the remarkable advancement in cold atom experiments and the possibility of probing fundamental theoretical models in the laboratory [4]. As a result, applications of numerical methods based on tensor states are getting increasingly common in descriptions of quantum gases in optical lattices [5 9]. Tensor states display an assortment of properties that can be exploited to absorb symmetries and implement transformations selectively rather that on the whole Hilbert space [10 12]. Nevertheless, in the present work tensor states are used as a tool that allow to put in practice the design outlined throughout the analysis that follows.
Let us introduce the Bose-Hubbard Hamiltonian in one dimension as follows

$$
\hat{H}=\sum_{j=1}^{N} \frac{U}{2} \hat{n}_{j}\left(\hat{n}_{j}-1\right)-J\left(\hat{a}_{j+1}^{\dagger} \hat{a}_{j}+\hat{a}_{j}^{\dagger} \hat{a}_{j+1}\right)+\mu_{j} \hat{n}_{j},
$$

being $\hat{n}_{j}=\hat{a}_{j}^{\dagger} \hat{a}_{j}$, while $\hat{a}_{j}$ and $\hat{a}_{k}^{\dagger}$ satisfy $\left[\hat{a}_{j}, \hat{a}_{k}^{\dagger}\right]=\delta_{j}^{k}$ and $\left[\hat{a}_{j}, \hat{a}_{k}\right]=0$ for $j, k=1,2, \ldots, N$. Operators $\hat{a}_{j}$ and $\hat{a}_{j}^{\dagger}$ represent the $j$ th mode. If these modes are associated to Wannier functions with no significant overlap in position [13, 14], as it is normally the case in applications of the Bose-Hubbard model, then $j$ numbers a site in a quantum chain. In order to allow for benchmarking against TEBD, open boundary conditions are embraced, $\hat{a}_{N+1}^{\dagger}=\hat{a}_{N+1}=0$, yet the validity of the central arguments here is not tied to such a specific kind of boundary conditions. Moreover, periodic boundary conditions were used in [3]. The Bose-Hubbard model undergoes a continuous phase transition from Mott insulator to Superfluid and its phase diagram as well as its phenomenology has been worked out in various contributions [15 17].

The standard version of TEBD makes use of the TrotterSuzuki Expansion (TSE) [18] to decompose the evolution operator as a product of transformations involving only nearest neighbor sites. This is done by splitting the evolution operator in two sub-evolutions, each generated by a part of the Hamiltonian grouping every other term of the sum in (11). The approach is efficacious, but it delimits the spectrum of applications to Hamiltonians with nearest-neighbor hopping or interaction, and open boundary conditions too. Here it is proposed to separate the Hamiltonian into single-particle and many-particle operators, such as they are understood from a secondquantization perspective, and then write the evolution operator as a composition of infinitesimal evolutions for each part. A second order split can thus be considered as follows

$$
e^{-i t_{s} \hat{H}} \approx e^{-i \frac{t_{s}}{2} \hat{H}_{M P}} e^{-i t_{s} \hat{H}_{S P}} e^{-i \frac{t_{s}}{2} \hat{H}_{M P}},
$$


knowing that

$$
\begin{gathered}
\hat{H}_{S P}=\sum_{j=1}^{N}\left(-\frac{U}{2}+\mu_{j}\right) \hat{n}_{j}-J\left(\hat{a}_{j+1}^{\dagger} \hat{a}_{j}+\hat{a}_{j}^{\dagger} \hat{a}_{j+1}\right), \\
\hat{H}_{M P}=\sum_{j=1}^{N} \frac{U}{2} \hat{n}_{j}^{2} .
\end{gathered}
$$

The only request being that the terms appearing in $\hat{H}_{M P}$, i.e., the interaction, must be local, but $\hat{H}_{S P}$ can take any form as long as it remains single-body. Under the action of single-particle transformations, it is known that the mode operators inherit the properties normally assigned to states and in particular a form of linearity that in turn induces a form of unitariety which governs the coefficients of the mode operators. It is this unitariety that will be important for the development of the current proposal and should be differentiated from the most common and general notion of unitariety.

Independently of the formulation of the exponential split, the simulation protocols that have relevance in this study employ the tensorial representation and the updating routines for one- and two-site transformations on a quantum chain that were first introduced in [19]. In coherence to this, the cost of a given computation is reported by the variable $\chi$, which establishes, in the shape of a polynomial, the maximum number of arithmetic operations necessary to calculate the action of an operator on at most two consecutive sites of the chain. It will be seen that all these elements play a role in the conception and testing of the methodologies that are the focus of this review.

In the next section the fundamentals of the technique, here referred to in general as mode folding (MF), are explained. Then it is seen how interaction is incorporated and how the whole proposal performs against characteristic parameters and in comparison to TSE. Subsequently, it is shown how to work with non-unitary transformations and the particularities found in the calculation of ground states. It is pointed out that different versions of the proposal can be used together in cases where ground state and evolution are both required, such as in studies of quench dynamics. Alternative approaches and conclusions are presented in the last part.

\section{MODE FOLDING}

A solution of the Schrodinger equation for a system of identical particles is given by

$$
|\psi(t)\rangle=\prod_{q=1}^{N} \frac{\left(\hat{\alpha}_{q}^{\dagger}\right)^{n_{q}}}{\sqrt{n_{q} !}}|0\rangle,
$$

where $\hat{\alpha}_{q}^{\dagger}(t)=e^{-i t \hat{H}} \hat{a}_{q}^{\dagger} e^{i t \hat{H}}(\hbar=1) . N$ is the number of modes and $M=\sum_{q=1}^{N} n_{q}$ is the total number of particles. It follows from Eq. (5) that $|\psi(0)\rangle=\prod_{q=1}^{N}\left|n_{q}\right\rangle$. If only single-particle transformations are considered $(U=0)$, then the evolution modes behave linearly (for the sake of clarity, time dependencies are stressed in some of the subsequent expressions)

$$
\hat{\alpha}_{q}^{\dagger}(t)=c_{1, q}(t) \hat{a}_{1}^{\dagger}+c_{2, q}(t) \hat{a}_{2}^{\dagger}+\ldots+c_{N, q}(t) \hat{a}_{N}^{\dagger} .
$$

The coefficients in this expression depend essentially on $\hat{H}_{S P}$ through a closed system of dynamical equations. Unitary operations on $|\psi(t)\rangle$ can be seen as transformations that act simultaneously on every $\hat{\alpha}_{q}^{\dagger}$. There are two types of such transformations that have applications in the folding method. The first one affects mode operators individually and is used to make the coefficients real. Such an effect is achieved by implementing the inverse of

$$
\hat{r}_{k}^{[l]}(t)=e^{i \phi_{l, k}(t) \hat{a}_{l}^{\dagger} \hat{a}_{l}}, \phi_{l, k}(t)=\arg c_{l, k}(t),
$$

getting as a result $c_{l, k} \hat{a}_{l}^{\dagger} \rightarrow\left|c_{l, k}\right| \hat{a}_{l}^{\dagger}$. When this is done consecutively for $l=1,2, \ldots, N$ only real coefficients are left in the equivalent of Eq. (6) with $q=k$. The $c_{l, k}$ 's can then be redefined as the absolute values of the original coefficients. The other type of transformation involves pairs of modes and is given by the inverse of

$$
\begin{gathered}
\hat{R}_{k}^{[j+1, j]}(t)=e^{i \theta_{j, k}(t) \hat{J}_{y}^{[j+1, j]}}, \\
\hat{J}_{y}^{[j+1, j]}=\frac{1}{2 i}\left(\hat{a}_{j+1}^{\dagger} \hat{a}_{j}-\hat{a}_{j}^{\dagger} \hat{a}_{j+1}\right) .
\end{gathered}
$$

The transformation is essentially a rotation since its generator is a genuine quantum angular momentum. The procedure yields

$$
\begin{array}{r}
\hat{R}_{k}^{[j+1, j]}{ }^{-1}\left(c_{j+1, q} \hat{a}_{j+1}^{\dagger}+c_{j, q} \hat{a}_{j}^{\dagger}\right) \hat{R}_{k}^{[j+1, j]}, \\
=c_{j+1, q}^{\prime} \hat{a}_{j+1}^{\dagger}+c_{j, q}^{\prime} \hat{a}_{j}^{\dagger}, \\
c_{j+1, q}^{\prime}=c_{j+1, q} \cos \left(\frac{\theta_{j, k}}{2}\right)-c_{j, q} \sin \left(\frac{\theta_{j, k}}{2}\right), \\
c_{j, q}^{\prime}=c_{j+1, q} \sin \left(\frac{\theta_{j, k}}{2}\right)+c_{j, q} \cos \left(\frac{\theta_{j, k}}{2}\right) .
\end{array}
$$

The fact that the change on the coefficients is unitary is pivotal to the ensuing discussion. After making $c_{j+1, q}^{\prime}=$ 0 it follows

$$
\tan \left(\frac{\theta_{j, q}}{2}\right)=\frac{c_{j+1, q}}{c_{j, q}}
$$

As a result, operator $\hat{a}_{j+1}^{\dagger}$ is neutralized in $\hat{\alpha}_{q}^{\dagger}$. In order to see the complete action of these operations, let us propose a view in which the evolved modes appear stacked as shown by (14). Transformations affect all the coefficients vertically aligned. In the first part of the proposed 
protocol, all the coefficients in the first row of the stack, i.e., those belonging to $\hat{\alpha}_{1}^{\dagger}$, are stripped from their complex phases employing transformations of the first kind. Notice that once this has been completed, all the coefficients in the stack have most likely changed, not just the ones in the first row. The next step consists in making $\hat{a}_{N}^{\dagger}$ disappear from the first row by operating on the two columns at the left of the stack, as indicated in (14), and then making $c_{N, 1}^{\prime}=0$. This cancellation technique is repeated systematically, advancing toward the right, until all the operators except $\hat{a}_{1}^{\dagger}$ are eliminated from the first row, as indicated by (15) and (16). It is worth noticing that the last of these operations entails the cancellation of all but the top $\hat{a}_{1}^{\dagger}$ in the column at the right extreme of the stack, by virtue of the unitariety of all previous transformations and the fact that the modes are orthonormal. At the end of this first series (or layer) of changes, the first mode has been folded and the stack of operators appears just like (17). The second layer of operations is aimed at folding the second mode and has a very similar structure, but it is carried without reaching the first mode, lest it unfolds. The scheme goes on in an orderly manner until the stack is left with a different operator in every level. At this point, it can be argued that the system has been returned to its initial state independently of the distribution of $n_{q}$ 's in Eq. (5).

$$
\begin{aligned}
& \mid \begin{array}{c|c}
\hat{R}_{1}^{[N, N-1]}{ }^{-1} & \\
\cline { 1 - 1 } c_{N, 1} \hat{a}_{N}^{\dagger}+c_{N-1,1} \hat{a}_{N-1}^{\dagger} & +c_{N-2,1} \hat{a}_{N-2}^{\dagger} \cdots+c_{1,1} \hat{a}_{1}^{\dagger} \\
c_{N, 2} \hat{a}_{N}^{\dagger}+c_{N-1,2} \hat{a}_{N-1}^{\dagger} & +c_{N-2,2} \hat{a}_{N-2}^{\dagger} \cdots+c_{1,2} \hat{a}_{1}^{\dagger} \\
\cdots & \cdots \\
c_{N, N} \hat{a}_{N}^{\dagger}+c_{N-1, N} \hat{a}_{N-1}^{\dagger} & +c_{N-2, N} \hat{a}_{N-2}^{\dagger} \cdots+c_{1, N} \hat{a}_{1}^{\dagger}
\end{array} \\
& \begin{array}{c|c|c|} 
& \hat{R}_{1}^{[N-1, N-2]}-1 & \\
\cline { 2 - 3 } c_{N, 2}^{\prime} \hat{a}_{N}^{\dagger}+ & c_{N-1,1}^{\prime} \hat{a}_{N-1}^{\dagger}+c_{N-2,1} \hat{a}_{N-2}^{\dagger} & \cdots+c_{1,1} \hat{a}_{1}^{\dagger} \\
\cdots & c_{N-1,2}^{\prime} \hat{a}_{N-1}^{\dagger}+c_{N-2,2} \hat{a}_{N-2}^{\dagger} & \cdots+c_{1,2} \hat{a}_{1}^{\dagger} \\
c_{N, N}^{\prime} \hat{a}_{N}^{\dagger}+ & c_{N-1, N}^{\prime} \hat{a}_{N-1}^{\dagger}+c_{N-2, N} \hat{a}_{N-2}^{\dagger} & \cdots+c_{1, N} \hat{a}_{1}^{\dagger}
\end{array}
\end{aligned}
$$

$$
\begin{array}{c|c}
c_{N, 2}^{\prime} \hat{a}_{N}^{\dagger}+\cdots+c_{3,2}^{\prime \prime} \hat{a}_{3}^{\dagger}+ & \begin{array}{c}
\hat{R}_{1}^{[2,1]}{ }^{-1} \\
c_{N, N}^{\prime} \hat{a}_{2}^{\dagger}+c_{1,1} \hat{a}_{1}^{\dagger} \\
c_{2,2}^{\prime} \hat{a}_{2}^{\dagger}+c_{1,2} \hat{a}_{1}^{\dagger} \\
\cdots \\
c_{N}^{\prime}+\cdots+c_{3, N}^{\prime \prime} \hat{a}_{3}^{\dagger}+ \\
c_{2, N}^{\dagger}+c_{1, N} \hat{a}_{1}^{\dagger}
\end{array} \mid \\
c_{N, 2}^{\prime} \hat{a}_{N}^{\dagger}+\cdots+c_{3,2}^{\prime \prime} \hat{a}_{3}^{\dagger}+c_{2,2}^{\prime \prime} \hat{a}_{2}^{\dagger} \\
\cdots \\
c_{N, N}^{\prime} \hat{a}_{N}^{\dagger}+\cdots+c_{3, N}^{\prime \prime} \hat{a}_{3}^{\dagger}+c_{2, N}^{\prime \prime} \hat{a}_{2}^{\dagger}
\end{array}
$$

Having determined the set of transformations required by this folding mechanism, it is possible to reassemble the evolved state implementing the sequence in reverse order. All of this can be mathematically synthesized as

$$
|\psi(t)\rangle=\prod_{k=N}^{1}\left(\prod_{l=k}^{N} \hat{r}_{k}^{[l]}(t) \prod_{j=k<N}^{N-1} \hat{R}_{k}^{[j+1, j]}(t)\right)|\psi(0)\rangle .
$$

However, Eq. (18) is not the only possibility. In particular, if the stack is folded starting by $\hat{\alpha}_{N}^{\dagger}$, it is found instead

$$
|\psi(t)\rangle=\prod_{k=1}^{N}\left(\prod_{l=N}^{N-k+1} \hat{r}_{k}^{[l]} \prod_{j=N-1}^{N-k+1<N} \hat{R}_{k}^{[j+1, j]}\right)|\psi(0)\rangle .
$$

Henceforth this latter approach will be referred to as the inverse $\mathrm{MF}$, in order to differentiate it from the former scheme, or "normal" MF. Although both forms are in principle equivalent, it will be seen that their numerics might display different accuracy.

\section{THE INCLUSION OF INTERACTION AND THE CASE OF RAPIDLY DECAYING COEFFICIENTS}

Since $|\psi(0)\rangle$ in Eqs. (18) and (19) can be any Fock state, it is possible to extend the formalism by writing an arbitrary initial state as a superposition of Fock states and then using the fact that the quantum evolution operator acts linearly on such a superposition. In order to incorporate interaction effects, the evolution operator is split, as in Eq. (2), into single-particle and many-particle step evolutions. The tensorial representation of the state is obtained applying the unitary transformations related to MF, which account for the single-particle step, as well as the many-particle step using the updating protocols demonstrated in Ref. [19]. Furthermore, because the interaction terms are assumed to be local, the latter step does not alter $\chi$, i.e., it does not produce changes in the size of the tensorial representation. To test the proposal, the obtained matrix product state (MPS) is passed to the Fock basis and then compared to benchmark results found from the diagonalization of the corresponding Hamiltonian in the Fock basis. In order to manage the error incurred by splitting the evolution operator, $t_{s}$ must be made small compared to the eigenvalues of the Hamiltonian. As a consequence, the distribution of coefficients in (14) is highly sparse, with most of the coefficients falling below machine accuracy. The exception being diagonal elements, which remain close to one, and their neighbors. As small coefficients can be a source of numerical errors, it is convenient to simply drop them and fold what is left of the stack. This leads to a slightly 

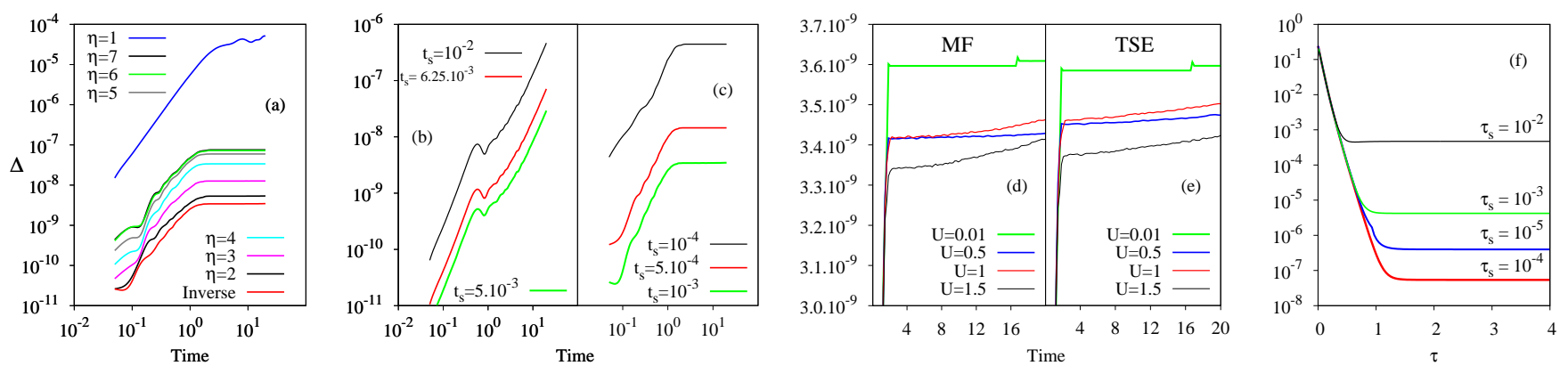

FIG. 1: Numerical error delivered by MF under different circumstances in boson chains of size $N=M=8$. (a) These calculations correspond to Hamiltonian (1) with $U=1, J=1, \mu=0$. In addition, $t_{s}=10^{-3}$ and $\chi=105$. (b)-(c) Inverse MF for different time slices. The simulated systems correspond to $U=1, J=1, \mu=0$, and $\chi=105$. (d)-(e) Error comparison between second order implementations of inverse MF and TSE for different $U$. In both cases $J=1, \mu=0, t_{s}=10^{-3}$ and $\chi=105$. (f) $\Delta=1-\left|\left\langle\psi_{G} \mid \psi(\tau)\right\rangle\right|^{2}$ for various values of $\tau_{s}$ in ground state calculations for $U=10, J=1, \mu=0$ and $\chi=\mathcal{O}(10)$. In all six graphs the initial state displays one boson at each site.

different version of Eq. (18), namely

$\left|\psi\left(t+t_{s}\right)\right\rangle=\prod_{k=N}^{1}\left(\prod_{l=k}^{k+\eta<N} \hat{r}_{k}^{l l]} \prod_{j=k<N}^{k+\eta-1<N-1} \hat{R}_{k}^{[j+1, j]}\right)|\psi(t)\rangle$,

where $\eta$ is the largest number of non-vanishing coefficients on either side of the stack diagonal. In order to measure the difference between the results delivered by MF and other reference results, the following error measure is introduced $\Delta=1-\left|\left\langle\psi^{\prime} \mid \psi\right\rangle\right|^{2}$, where $|\psi\rangle^{\prime}$ comes from a tensorial representation and $|\psi\rangle$ from a standard matricial analysis. It can be seen in figure1(a) that different versions of MF produce results with distinct accuracies. It can also be seen that the best estimation is given by the inverse MF, although the difference with the next best estimation is rather marginal. The observed optimal parameter is $\eta=2$, but in general the appropriate $\eta$ depends on the distribution of coefficients in (14). For small values of $t_{s}$ the distribution is tight and the optimal $\eta$ is short. Likewise, $\eta$ should grow with increasing $t_{s}$. It is empirically seen that the best performance is achieved when the smallest considered coefficient is approximately of the same order of magnitude than the square root of machine accuracy. The reduction in correctness observed when more coefficients are included occurs because substantial error is transmitted to some folding angles calculated using coefficients with an insufficient number of significant figures at the beginning of each layer of calculations. Contrariwise, no such a tendency is observed in the inverse scheme since, even though poorly estimated coefficients are retained, the folding routine starts by the biggest coefficients on each layer. This makes inverse MF reliable, although it requires the inclusion of all the folding operations. Normal folding taking less coefficients is numerically more efficient, but a little bit less exact. Figures 1(b) and1(c) show the characteristic behavior of the error as a function of time for different values of the time slice. For relatively large $t_{s}, \Delta$ 's growth is essen- tially polynomial. As $t_{s}$ diminishes, the corresponding error curves scale down until for an optimal time slice the error starts showing saturation. As $t_{s}$ diminishes further, the error scales up but the saturation profile remains. It can therefore be argued that while the theoretical error is gradually suppressed with decreasing $t_{s}$, the computing error caused by division by small numbers augments. Similarly, the optimal value $\chi=105$ seems to be independent of the values given to $\eta$ and $t_{s}$. However, the dependence of the error with respect to $\chi$ is rather characteristic. Making $\chi$ slightly smaller than its optimal value produces exponentially growing deviations from the actual state at very early times. This contrasts with the behavior of $\Delta$ in simulations where although the time slice has been chosen well above its optimal value, $\chi$ has remained at or above its equilibrium value. In such cases the error growth is polynomial and the simulation can be sustained for quite longer intervals. In this sense it can be said that a right estimation of $\chi$ is more important for numerics than the chosen $t_{s}$. This feature is encountered in both MF and TSE simulations. The comparative graphs depicted in figures 1(d) and 1(e) suggest that the difference in terms of deviations from the correct state between MF and TSE is minimal over a wide range of values of the ratio $U / J$. Since it has been shown in Ref. [3] that folding alone delivers results with a tolerance of the order of machine accuracy, it follows that the observed error is mostly due to the splitting of the evolution operator into single- and many particle parts and that such an error is comparable to the one produced by TSE. In terms of efficiency, because both approaches seem to require the same $\chi$, the determinant factor is the number of transformations effectuated on pairs of modes per loop in a computer routine, i.e., the number of updates of the tensorial representation necessary to advance the state a time $t_{s}$. In a second order version such a number is approximately $3 N / 2$ for TSE and $N \eta-\eta(\eta+1) / 2$ for MF. The difference can be understood in terms of the 
cost associated to the inclusion of more hopping terms in $\mathrm{MF}$, in contrast to the less-strict neighbor approach dictated by TSE.

\section{NON-UNITARY TRANSFORMATIONS AND THE CALCULATION OF GROUND STATES}

Ground states play a prominent role in the study of quantum systems and it is known that they usually display less entanglement than dynamical states. As a consequence, the associated simulation cost in terms of tensor states is manageable to a great extent. Simulation protocols that rely on MPS can be formulated as variational methods. This is the case of DMRG, where the ground state is found as the network that minimizes the energy. Another approach is to realize the limit

$$
\left|\psi_{G}\right\rangle=\lim _{\tau \rightarrow \infty} \frac{e^{-\tau \hat{H}}\left|\psi_{0}\right\rangle}{\| e^{-\tau \hat{H}}\left|\psi_{0}\right\rangle \|},
$$

as an iteration of an infinitesimal imaginary-time evolution of length $\tau_{s}$. Such is the strategy followed in the context of TEBD and it is also the approach adopted here. The crux of the problem is that imaginary-time evolutions are not unitary, ergo the foundation of the MPS updating method [20] and the folding technique is compromised. To deal with the issue it is important, on the one hand, to use a sufficiently small "imaginary" time slice $\tau_{s}$, so that the corresponding advancement is close enough to unitary and the updating protocols work. On the other hand, it is necessary to find a folding protocol that is not as heavily dependent on the unitariety of the evolution operation. Moreover, the protocol itself must integrate genuine non-unitary transformations so as to account for the non-unitariety of the whole transformation. In accordance with these premises, let us introduce

$$
\hat{Q}^{[j+1, j]}=e^{\epsilon_{j} \hat{j}_{x z}^{[j+1, j]}},
$$

where,

$$
\begin{gathered}
\hat{J}_{x z}^{[j+1, j]}=\cos \varphi_{j} \hat{J}_{x}^{[j+1, j]}+\sin \varphi_{j} \hat{J}_{z}^{[j+1, j]}, \\
\hat{J}_{x}^{[j+1, j]}=\frac{1}{2}\left(\hat{a}_{j+1}^{\dagger} \hat{a}_{j}+\hat{a}_{j}^{\dagger} \hat{a}_{j+1}\right), \\
\hat{J}_{z}^{[j+1, j]}=\frac{1}{2}\left(\hat{a}_{j+1}^{\dagger} \hat{a}_{j+1}+\hat{a}_{j}^{\dagger} \hat{a}_{j}\right) .
\end{gathered}
$$

Transformation (22) depends on $\epsilon_{j}$, which is supposed to be small, and also on $\varphi_{j}$, which can take any value in $[0,2 \pi]$. The operation is defined in terms of the quantum angular momenta $\hat{J}_{x}$ and $\hat{J}_{z}$ but it is not strictly a rotation. The action is confined to the $j$ th and $j+1$ th modes and the effect of the inverse on the coefficients of the $q$ th row is

$$
\begin{gathered}
c_{j+1, q}^{\prime}=-c_{j, q} \cos \varphi_{j} \sinh \left(\frac{\epsilon_{j}}{2}\right)+ \\
c_{j+1, q}\left(\cosh \left(\frac{\epsilon_{j}}{2}\right)-\sin \varphi_{j} \sinh \left(\frac{\epsilon_{j}}{2}\right)\right), \\
c_{j, q}^{\prime}=-c_{j+1, q} \cos \varphi_{j} \sinh \left(\frac{\epsilon_{j}}{2}\right)+ \\
c_{j, q}\left(\cosh \left(\frac{\epsilon_{j}}{2}\right)+\sin \varphi_{j} \sinh \left(\frac{\epsilon_{j}}{2}\right)\right) .
\end{gathered}
$$

Demanding that both $c_{j+1, q}^{\prime}=0$ and $c_{j, q+1}^{\prime}=0$, it results

$$
\begin{aligned}
\tan \varphi_{j} & =\frac{c_{j+1, q} c_{j+1, q+1}-c_{j, q} c_{j, q+1}}{2 c_{j+1, q} c_{j, q+1}}, \\
\tanh \left(\frac{\epsilon_{j}}{2}\right) & =\frac{c_{j+1, q}}{c_{j+1, q} \sin \varphi_{j}+c_{j, q} \cos \varphi_{j}} .
\end{aligned}
$$

It can be seen that the hyperbolic tangent is well defined as long as $\left|c_{j+1, q}\right| \ll\left|c_{j, q}\right|$ and $\left|c_{j, q+1}\right| \ll\left|c_{j+1, q+1}\right|$, except if $c_{j+1, q}=0$ or $c_{j, q+1}=0$. These consistency conditions are met if $\tau_{s}$ is sufficiently short but non-vanishing. In particular, $\tau_{s}$ can be set to a value for which $\eta=1$, yielding the operator stack shown in (30). In this context the coefficients are most likely real and positive, if they are not, they can be adjusted using unitary operations as previously discussed. A two-mode transformation is applied on the right-extreme of the stack, resulting in the simultaneous cancellation of $\hat{a}_{2}^{\dagger}$ in the first row and $\hat{a}_{1}^{\dagger}$ in the second row 21], leaving the stack as indicated in (31). The lack of unitariety of (31) means coefficient $c_{1,1}^{\prime}$ can be hardly equal to one, hence it is normalized using the inverse of $\hat{q}^{[j]}=e^{\delta_{j} \hat{a}_{j}^{\dagger} \hat{a}_{j}}$, with $\delta_{j}=\log c_{j, j}^{\prime \prime}$, when $j=1$. Thereafter the first mode is folded and the cancellationand-normalization sequence starts over again as depicted by (31) and (32).

$$
\begin{aligned}
& \begin{array}{r|l|} 
& \multicolumn{1}{|c}{\hat{Q}^{[2,1]}{ }^{-1}} \\
\cline { 2 - 2 } c_{3,2} \hat{a}_{3}^{\dagger}+ & c_{2,1} \hat{a}_{2}^{\dagger}+c_{1,1} \hat{a}_{1}^{\dagger} \\
c_{2,2} \hat{a}_{2}^{\dagger}+c_{1,2} \hat{a}_{1}^{\dagger} \\
c_{4,3} \hat{a}_{4}^{\dagger}+c_{3,3} \hat{a}_{3}^{\dagger}+ & c_{2,3} \hat{a}_{2}^{\dagger} \\
c_{5,4} \hat{a}_{5}^{\dagger}+c_{4,4} \hat{a}_{4}^{\dagger}+c_{3,4} \hat{a}_{3}^{\dagger} & \\
. &
\end{array} \\
& \begin{array}{r|c|c|} 
& \hat{Q}^{[3,2]}{ }^{-1} & \hat{q}^{[1]}{ }^{-1} \\
\cline { 2 - 3 } c_{4,3} \hat{a}_{4}^{\dagger}+ & c_{3,2} \hat{a}_{3}^{\dagger}+c_{2,2}^{\prime} \hat{a}_{2}^{\dagger} & c_{1,1}^{\prime} \hat{a}_{1}^{\dagger} \\
c_{3,3} \hat{a}_{3}^{\dagger}+c_{2,3}^{\prime} \hat{a}_{2}^{\dagger} & \\
c_{5,4} \hat{a}_{5}^{\dagger}+c_{4,4} \hat{a}_{4}^{\dagger}+ & c_{3,4} \hat{a}_{3}^{\dagger} & \\
. & &
\end{array} \\
& \begin{array}{c|c|c|}
c_{5,4} \hat{a}_{5}^{\dagger}+ & \hat{Q}^{[4,3]}{ }^{-1} & \hat{q}^{[2]}{ }^{-1} \\
\cline { 2 - 2 } & & \\
& c_{4,3} \hat{a}_{4}^{\dagger}+c_{3,3}^{\prime} \hat{a}_{3}^{\dagger} & \hat{a}_{2}^{\dagger} \\
c_{4,4} \hat{a}_{4}^{\dagger}+c_{3,4}^{\prime} & & \\
& &
\end{array}
\end{aligned}
$$




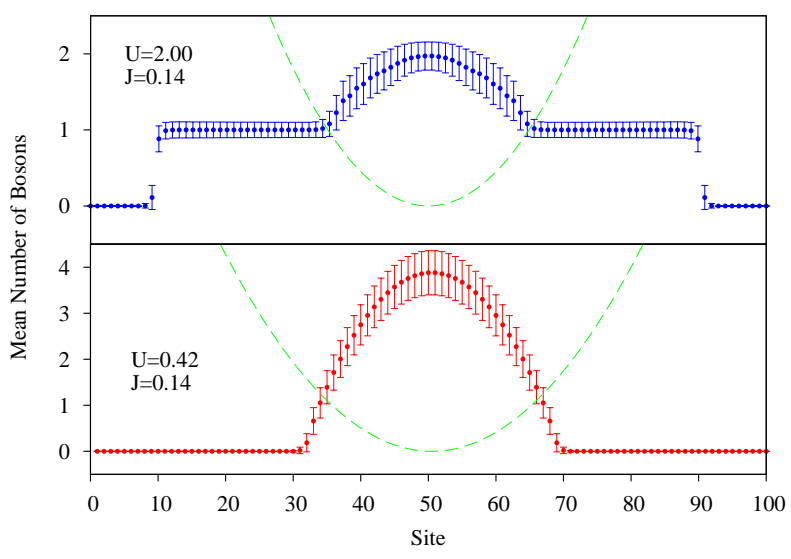

FIG. 2: Mean number of particles and fluctuations (error bars) for the ground state of a boson chain with $N=M=100$ and open boundary conditions. In both cases the confining potential is $\mu_{j}=0.0046(j-50.5)^{2}$ (dashed line). Additionally, $\tau_{s}=10^{-5}$ and $\chi=\mathcal{O}(10)$.

Gathering all the involved steps and putting them in reverse order, the advanced state can be written as

$$
\left|\psi\left(\tau+\tau_{s}\right)\right\rangle=\left(\prod_{j=N-1}^{1} \hat{Q}^{[j+1, j]} \hat{q}^{[j]}\right) \hat{q}^{[N]}|\psi(\tau)\rangle .
$$

Furthermore, interaction mechanisms can be incorporated using the split-operator method, analogously to the case of normal evolution. Beginning with a state that has an overlap with the ground state, the whole set of operations is applied systematically until a convergence criterion is satisfied. Figure 1(f) depicts the convergence behavior as captured by measuring the fidelity to the actual ground state as a function of $\tau$. For the cases studied, convergence is steady but tolerance depends on $\tau_{s}$. As seen for real time calculations, there exists an optimal slice that minimizes the simulation error. The test simulations displayed in figure 2 show a system of interacting bosons subject to a confining potential under the regimes of Mott Insulator (top) and Superfluid (bottom). Both phases can be realized in experiments of cold atoms in optical lattices with the parameters used in the plots of figure 2. These outcomes coincide qualitatively with the results obtained in [22] using TSE in chains with the same set of parameters and size. Of relevance is the fact that, as long as $\chi$ is optimal, the symmetry of the state is correctly reproduced by the folding method in spite of the relatively large number of operations correlating modes that are distant. All this reaffirms that little error is genrated by the folding process alone, even in the presence of interaction. One advantage of MF is that realand imaginary-time versions can be used in conjunction to simulate quench dynamics where the initial state is set to the ground state of a Hamiltonian with dominant interaction. This is especially useful when the evolution
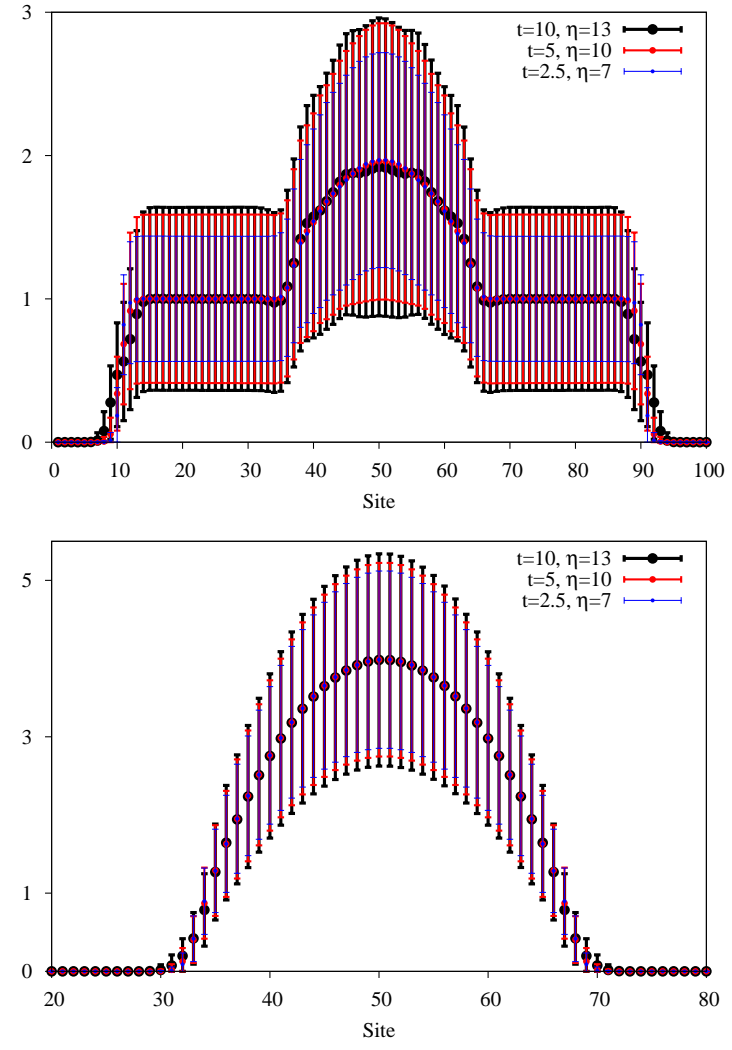

FIG. 3: Mean number of particles and fluctuations after a quench. Once interaction and trapping have been shut down, $U=\mu=0$, the dynamics is generated only by the kinetic term $J=0.14$. The simulation constant is $\chi=\mathcal{O}\left(10^{3}\right)$. The corresponding states at $t=0$ are shown in figure 2 Mean values do not change much, rather, it is fluctuations that capture the state evolution.

is governed by a single-particle Hamiltonian. Figure 3 exemplify this approach using the ground states featured in figure 2 as initial states. After interaction and confining potential have been switched off, atoms evolve freely toward a fluctuation-dominated phase. In the time span considered, the atom cloud does not expand significantly beyond its initial domain, instead, there is an increase in hopping across the whole extent of the cloud. As longer times are addressed, the simulation cost soars.

The techniques introduced in this work make use of the basic idea of folding of modes to develop numerical routines that calculate the quantum state. Although each application is different, the essence of every method is the same. Certainly, the folding idea is potentially versatile and may give rise to additional simulation protocols. To give an example of another application, let us conceive the dynamical modes as a superposition of the 
Hamiltonian eigenvectors as follows

$$
\left(\begin{array}{c}
\alpha_{1}^{\dagger}\left(t_{s}\right) \\
\alpha_{2}^{\dagger}\left(t_{s}\right) \\
\alpha_{3}^{\dagger}\left(t_{s}\right) \\
\vdots
\end{array}\right)=\hat{b}_{1}^{\dagger} e^{-i t_{s} E_{1}}\left(\begin{array}{c}
E_{11} \\
E_{21} \\
E_{31} \\
\vdots
\end{array}\right)+\hat{b}_{2}^{\dagger} e^{-i t_{s} E_{2}}\left(\begin{array}{c}
E_{12} \\
E_{22} \\
E_{32} \\
\vdots
\end{array}\right)+\ldots
$$

so that

$$
\hat{b}_{q}^{\dagger}=\hat{a}_{1}^{\dagger} E_{1 q}+\hat{a}_{2}^{\dagger} E_{2 q}+\hat{a}_{3}^{\dagger} E_{3 q}+\ldots
$$

Initially, making $t_{s}=0$, the superposition lacks the exponential terms. Therefrom, the first move is to fold $\hat{b}_{1}^{\dagger}$ using unitary transformations until only $\hat{a}_{1}^{\dagger}$ remains. Without further action, $\hat{a}_{1}^{\dagger}$ is automatically expelled from the updated versions of all the other $\hat{b}$ 's. Next, the singleparticle operation $e^{-i t_{s} E_{1} \hat{a}_{1}^{\dagger} \hat{a}_{1}}$ is applied. The inverses of the transformations employed to fold $\hat{b}_{1}^{\dagger}$ are then executed in reverse order. This brings $\hat{b}_{1}^{\dagger}$ back into the superposition, but accompanied by the term $e^{-i t_{s} E_{1}}$, leaving the other $\hat{b}$ 's as they were at the beginning. An analogous sequence is then applied, focusing this time on $\hat{b}_{2}^{\dagger}$, in order to incorporate $e^{-i t_{s} E_{2}}$. The rest of the protocol ensues in a logical manner until all the exponentials are introduced. The transformations are then collected and put together to assemble an evolution operator that can be implemented in terms of tensor states. A very similar scheme can be formulated to handle non-unitary transformations. The problem is that the corresponding numerics is not very stable owing to the recurrent folding-unfolding sequence, but the proposal is perfectly valid. Additional folding schemes will be reported in future contributions.

\section{CONCLUSIONS}

Numerical techniques with applications to interacting systems have been introduced and probed. The proposals are developed in the context of local interactions and are based on the idea of mode folding and on the efficient use of tensor states. Real as well as imaginary time implementations of the evolution operator are discussed, elaborating on the handling of each particular case. The method compares well with TSE in terms of accuracy and simulation time. However, its potential mainly resides, on the one hand, in the possibility of managing hopping of arbitrary scope, and on the other hand, in the perspective that it offers about the role of single-body- and many-body structures in the calculation of a quantum state. As an extension, it would be interesting to consider the prospects of folding of fermionic modes. As it is known, the sign-problem prevents the effective use of TSE. It remains to be seen whether the alternative path taken by MF somehow allows to circumvent the adverse effects of fermion algebra.

[1] U. Schollwock, Annals of Phys. 32696 (2011).

[2] R. Orus, Ann. Phys. 349 117-158 (2014).

[3] J. Reslen, Mex. J. Phys. (RMF) 59482 (2013).

[4] J.P. Ronzheimer, M. Schreiber, S. Braun, S.S. Hodgman, S. Langer, I.P. McCulloch, F. Heidrich-Meisner, I. Bloch and U. Schneider, Phys. Rev. Lett. 110205301 (2013). C. D'Errico, E. Lucioni, L. Tanzi, L. Gori, G. Roux, I.P. McCulloch, T. Giamarchi, M. Inguscio and G. Modugno, arXiv:1405.1210

[5] M. Lacki, B. Damski and J. Zakrzewski, Phys. Rev. A 89033625 (2014).

[6] M. Piraud, Z. Cai, I.P. McCulloch and U. Schollwock, Phys. Rev. A 89063618 (2014).

[7] I. Danshita, Phys. Rev. Lett. 111025303 (2013), I. Danshita and C.W. Clark, Phys. Rev. Lett. 102030407 (2009).

[8] J. Reslen and S. Bose, Phys. Rev. A, 80012330 (2009).

[9] S. Sorg, L. Vidmar, L. Pollet and F. Heidrich-Meisner, arXiv:1405.5404

[10] A.E. Feiguin, R.D. Somma and C.D. Batista, Phys. Rev. B 88075145 (2013).

[11] S. Singh, R.N.C. Pfeifer, G. Vidal and G. K. Brennen, Phys. Rev. B 89075112 (2014). S. Singh, H.Q. Zhou and G. Vidal, New J. Phys. 12033029 (2010).

[12] T.H. Johnson, S.R. Clark and D. Jaksch, Phys. Rev. E 82036702 (2010).

[13] R. Walters, G. Cotugno, T.H. Johnson, S.R. Clark and D. Jaksch, Phys. Rev. A 87043613 (2013).

[14] M.A. Cazalilla, R. Citro, T. Giamarchi, E. Orignac and M. Rigol, Rev. Mod. Phys. 83, 1405-1466 (2011).

[15] M.P.A. Fisher, P.B. Weichman, G. Grinstein and D.S. Fisher, Phys. Rev. A, 40546 (1989).

[16] D. Rossini and R. Fazio, New J. Phys. 14065012 (2012). L. Urba, E. Lund and A. Rosengren, J. Phys. B: At. Mol. Opt. Phys. 395187 (2006).

[17] M. Foss-Feig and A.M. Rey, Phys. Rev. A 84053619 (2011).

[18] I. Dhand and B.C Sanders, J. Phys. A: Math. Theor. 47 265206 (2014).

[19] G. Vidal, Phys. Rev. Lett. 91, 147902 (2003).

[20] R. Orus and G. Vidal, Phys. Rev. B 78, 155117 (2008).

[21] In addition, this operation turns on $\hat{a}_{1}^{\dagger}$ in the third row, but with a coefficient that is below machine accuracy.

[22] J. Reslen, Ph.D Thesis. arXiv:1002.4001 pp. 79. 